\title{
[Fe/H] RATIOS IN POPULATION II STARS
}

\author{
R. EARLE LUCK \\ Department of Astronomy \\ Case Western Reserve University \\ Cleveland, $\mathrm{OH} 44106$ \\ USA
}

\begin{abstract}
Abundances of Population II stars of the field and globular cluster systems are reviewed. Emphasis is placed on $[\mathrm{Fe} / \mathrm{H}]$ ratios determined from analyses of moderate- and high-resolution spectroscopic data. The status of analyses of metal-deficient stars is examined from the viewpoint of number of analyses and number of stars, overall distribution of the $[\mathrm{Fe} / \mathrm{H}]$ ratios, and systematics of the $[\mathrm{Fe} / \mathrm{H}]$ ratios.
\end{abstract}

\section{Introduction}

One of the more challenging problems in the field of Population II abundances is to define what is meant by Population II. A simplistic view is to define Population II as those stars which are roughly coeval with the earliest star formation epochs in the galaxy and thus were metal poor. The concept of a multicomponent galaxy: halo, thick disk, thin disk, and a nuclear bulge, has made the strict Population II concept of a single metal-poor population untenantable. For the purpose of this review, Population II will be defined without regard to kinematics and solely on the basis of the $[\mathrm{Fe} / \mathrm{H}]$ ratio. The operant definition is: $[\mathrm{Fe} / \mathrm{H}]$ $<-0.75$ is a Population II star. This level of abundances is somewhat larger than generally used in discussions of this type $([\mathrm{Fe} / \mathrm{H}]<-1.0$ is more typical) but allows us to include metal-rich globular clusters and stars of the thick disk. Since the definition is by metallicity, metal-poor dwarfs are also included.

Heavy-element abundances in Population II stars have been examined for the past thirty years for clues about the chemical evolution of the galaxy. Initially, these studies concentrated on determination of $[\mathrm{Fe} / \mathrm{H}]$ ratios as iron, in particular $\mathrm{Fe} \mathrm{I}$, has many lines making an abundance determination somewhat easier and hopefully, more certain. In the past decade more emphasis has been placed on the determination of $[\mathrm{M} / \mathrm{Fe}]$ ratios where $M$ is a metal of interest. While the $[\mathrm{M} / \mathrm{Fe}]$ ratios contain more astrophysical information, especially concerning sites of nucleosynthesis, the $[\mathrm{Fe} / \mathrm{H}]$ ratio is still a fundamental quantity. In some cases it is the only abundance which can be determined; while for most objects, it is still the abundance with the smallest (formal) error bar.

Abundance trends in Population II field and cluster objects have been reviewed thoroughly a number of times in the past 5 years: see especially Spite and Spite (1985) and Wheeler, Sneden, and Truran (1989) as well as the compendium volume edited by Cayrel de Strobel, Spite, and Lloyd Evans (1988). As the state of knowledge has not increased dramatically in the time since these reviews, I shall concentrate on a discussion of other aspects of the analyses: first a census of the analyses, followed by a discussion of systematics within the analyses. 


\section{Census}

The $[\mathrm{Fe} / \mathrm{H}]$ ratios to be inspected have been derived from analyses of moderate- to high-resolution $(\Delta \mathrm{U} \leq 0.5$ $\AA$ ) spectroscopic data. As the current topic is $[\mathrm{Fe} / \mathrm{H}]$ ratios, only those analyses that have derived (hopefully) independent values have been included; that is, analyses which quote $[\mathrm{Fe} / \mathrm{H}]$ ratios have not been included in the bibliographic database. For work previous to 1984 , the dominant source of reference material is the $[\mathrm{Fe} / \mathrm{H}]$ catalog of Cayrel de Strobel et al. (1985) (the original reference is cited in the database -not the catalog). For 1984 to the present an independent search of the literature was performed (guided by SIMBAD references for individual field stars and globular clusters). The number of references containing appropriate $[\mathrm{Fe} / \mathrm{H}]$ data was 141 , distributed by year of publication as shown in Figure 1. In the context of this discussion an "analysis" will be taken to mean the total contents of a published work which usually includes multiple stars. This data is not subdivided by cluster versus field as a significant number of papers give data for both types of objects. The significant increase in the number of analyses about 1980 reflects large optical telescopes coming on-line in the mid-1970's. The decline in the number of analyses after 1982 reflects not a decline in interest in metal-poor stars but rather a trend towards including more stars per analysis. The number of stars analyzed per year is shown in Figure 2 and it is clear that 1981 was a high point in the determination of metal-poor abundances but that since that time the number of stars analyzed per year has generally been increasing. As regards the type of stars (cluster versus field and within the field red giants versus dwarfs) being analyzed, there has been little change as a function of time. As shown by Figure 2, the number of cluster stars analyzed has not varied greatly from year-to-year since the late 1970's, indicating a constant effort to increase the quantity and quality of the analyses. Among the field stars there has been a constant effort since the early 1970's to determine $[\mathrm{Fe} / \mathrm{H}]$ ratios for metal-poor dwarfs. The larger part of the work on the red-giants coincides with that on the cluster stars (and not coincidentally comes soon after with the publication of the first realistic models of metal-poor red giants: Gustafsson et al. (1975)). The essence of this discussion is that we have not altered the thrust of this type of research: we are still analyzing the same types of stars in about the same relative numbers but are increasing the number of stars analyzed of each type with (hopefully) increased accuracy. 
How many stars have been analyzed and what are their overall properties? Within the 141 references found there are 750 analyses of 404 individual metal-poor stars. Of these 404 objects 210 are field stars and 194 are cluster stars. In Table 1 some global properties of the sample are shown - it must be emphasized that the means in the table have been taken over all stars in the subgroups; i.e., the cluster data have all been combined and real differences in the compositions of clusters ignored. For the field the data in addition to being shown as a whole have also been separated into the groups by gravity roughly corresponding to metal-poor red giants, intermediate-type stars, and dwarfs.

\begin{tabular}{|c|c|c|c|c|c|}
\hline \multicolumn{6}{|c|}{ Table 1 } \\
\hline & $\begin{array}{c}\text { Globular } \\
\text { Clusters }\end{array}$ & \multicolumn{5}{c|}{ Field Stars } \\
\cline { 3 - 6 } & & Total & $\lg \mathrm{g}<2.0$ & $2 \leq \lg \mathrm{g} \leq 3.5$ & $\lg \mathrm{g}>3.5$ \\
\hline $\mathrm{N}$ & 194 & 210 & 75 & 48 & 87 \\
\hline$\left\langle\mathrm{T}_{\mathrm{eff}}\right\rangle$ & 4210 & 5410 & 4390 & 5600 & 6180 \\
\hline$\langle\mathrm{Log} \mathrm{g}\rangle$ & 1.03 & 2.70 & 0.97 & 2.59 & 4.25 \\
\hline$\langle[\mathrm{Fe} / \mathrm{H}]\rangle$ & -1.40 & -1.62 & -1.80 & -1.63 & -1.45 \\
\hline
\end{tabular}

The metallicity distribution of the total sample is shown in histogram form in Figure 3. The abundances used for cases in which there are multiple analyses are simple

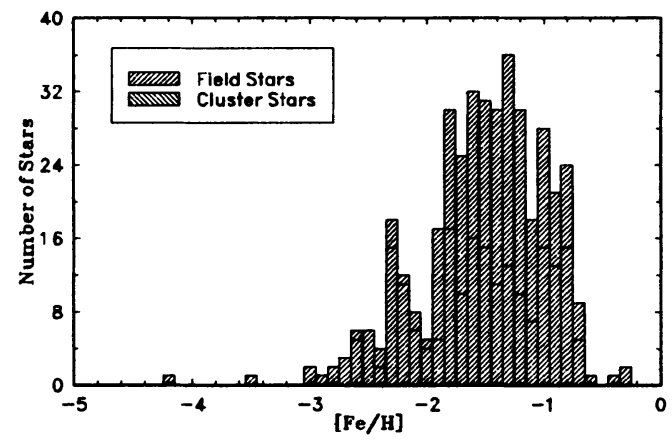

Figure 3: The cumulative $[\mathrm{Fe} / \mathrm{H}]$ distribution from the field and cluster metal-poor stars. rather similar if the $[\mathrm{Fe} / \mathrm{H}]<-2.2$ region is ignored. This result needs an in depth analysis of its statistical significance and bias. If these overall distributions do turn out to be the same this has great significance for galactic chemical evolution. 


\section{Systematics of the Analyses}

Evaluation of the quality of the $[\mathrm{Fe} / \mathrm{H}]$ data for metal-poor stars is a difficult proposition. The data used varies in resolution and signal-to-noise. The methods vary from "simple" curve-of-growth techniques to spectrum synthesis. The physical data (gf values, damping constants, etc.) vary from analysis to analysis (as well as from person to person: there is a personal equation in abundances). The only way to evaluate analyses is on a case by case basis and even then it is difficult as the usual case is that only the internal uncertainty can be evaluated directly. The real difficulty lies in the question of systematics in the abundances. This question will be returned to in the next section.

A number of the stars in the database have been analyzed multiple times. One would hope that as the number of analyses increases that there would be some convergence in the determination of the $[\mathrm{Fe} / \mathrm{H}]$ ratio. A manifestation of such a convergence would be that as the number of independent analyses increases, the standard deviation of those individual determinations about the mean abundance should decrease. In Figure 4 the standard deviation of the $[\mathrm{Fe} / \mathrm{H}]$ ratio is plotted versus the number of analyses. Let us emphasize that this standard deviation is not the internal uncertainty of the individual determinations but is a measure of spread in $[\mathrm{Fe} / \mathrm{H}]$ of the analyses themselves. Unfortunately, as Figure 4 makes apparent, our naive expectation concerning the behavior of the standard

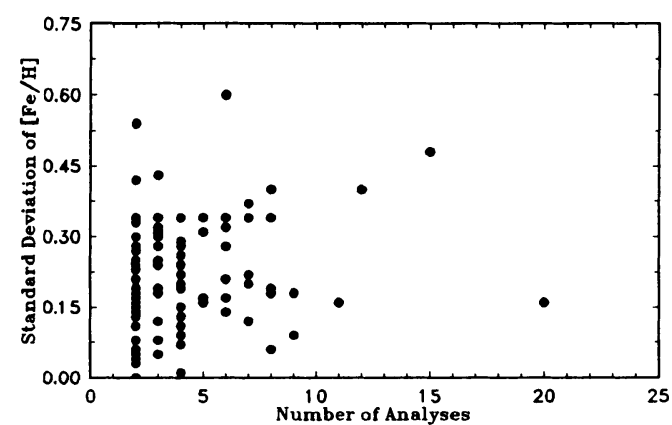

Figure 4: The standard deviations of the $[\mathrm{Fe} / \mathrm{H}]$ ratios for individual stars with multiple $[\mathrm{Fe} / \mathrm{H}]$ determinations. deviation is not born out in practice. The hoped for result would be that the plot would be a lower triangular figure indicating that the standard deviation is decreasing. However, the scatter in the standard deviation remains essentially constant indicating that there has been no convergence in the determination of the $[\mathrm{Fe} / \mathrm{H}]$ ratio with increasing numbers of analyses.

To further examine the systematics of the abundances, the behavior of $[\mathrm{Fe} / \mathrm{H}]$ as a function of effective temperature and gravity for both cluster and field stars has been investigated. There is no relation apparent between these quantities for the total sample nor for the cluster or field stars taken separately. Two quantities which are related are effective temperature and gravity (see Figure 5). This relation represents an admixture of masses and luminosities and is a "selection" effect: metal-poor red-giants are by definition cool and have low gravity while the dwarfs selected for analysis tend to be hotter and have higher gravity. It would be of interest to expand the sample by analysis of cool dwarfs (which do exist in large numbers but which are very faint) and higher temperature low gravity objects. The latter objects do have a few representatives in Figure 5 but these objects tend to be rather peculiar: i.e., they are high-latitude supergiants which often have anomalous CNO abundances.

The data shown in Table 1 indicates that in the field stars the low gravity objects have a lower mean metallicity than the higher gravity objects. As Figure 5 shows that the temperature and gravity are correlated, it would seem that abundance and temperature should correlate for the field stars (which have the greatest range in effective temperature). 


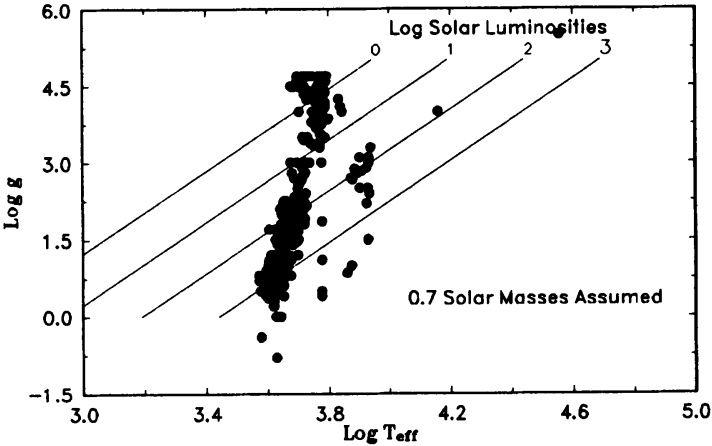

Figure 5: $\log \mathrm{T}_{\text {eff }}$ versus $\log \mathrm{g}$ for the program stars. The labelled lines are tracks in $\log \mathrm{T}_{\text {eff }}-\log \mathrm{g}$ for various luminosities at constant mass.

The previous paragraph asserted this to not be the case. The explanation is that stars with $[\mathrm{Fe} / \mathrm{H}]<-2.0$ are essentially uniformly distributed in temperature (but not gravity) but that at the hotter temperatures there are proportionately somewhat more stars with $[\mathrm{Fe} / \mathrm{H}]$ $>-2.0$.

\section{How accurate are the individual analyses?}

The metal-poor star with the largest number of analyses (20) is HD 122563 (HR 5270). Table 2 presents the range of values derived for this star. Unfortunately, ranges of this size are not uncommon in abundance analyses when results from different researchers are compared.

\begin{tabular}{||c|c|c|c|c||}
\hline \multicolumn{5}{|c|}{ Table 2 } \\
\hline & Minimum & Maximum & Mean & $\sigma$ \\
\hline $\mathrm{T}_{\mathrm{eff}}$ & 4500 & 4700 & 4570 & 135 \\
\hline $\log \mathrm{g}$ & 0.6 & 1.5 & 1.1 & 0.3 \\
\hline$[\mathrm{Fe} / \mathrm{H}]$ & -2.93 & -2.35 & -2.61 & 0.16 \\
\hline
\end{tabular}

Given the number of analyses of HD 122563, it is of interest to determine if there are any systematic trends in time for the results; i.e., the star has most likely not changed but have we? In this context we wish to know if improvements in observational or physical data have lead to a convergence in parameters and metallicity. In Figure 6 the individual quantities that went into Table 2 are plotted against date of publication and there are a number of comments which can be made. First, there is no indication of a systematic trend in the effective temperature. In terms of the gravity, there is a downward trend from 1972 through 1983 after which the derived gravities increased by nearly an order of magnitude and held constant until 1990 when it reverted back to the 1983 value! During this period the $[\mathrm{Fe} / \mathrm{H}]$ ratio showed an essentially linear increase until 1990 when it experienced a precipitous decline! What is going on in these analyses? Are the assumptions behind the analysis incorrect, is the observational data suspect, could the physical data be incorrect? is is likely that to some degree all of the above enter into the explanation. As this review is most concerned with the $[\mathrm{Fe} / \mathrm{H}]$ ratio, let us investigate that quantity to determine what is necessary for the determination of a reliable answer.

In order to determine a reliable $[\mathrm{Fe} / \mathrm{H}]$ ratio it is necessary to have reliable stellar parameters. Given the range in effective temperature and especially in gravity shown above for HD 122563 it is difficult to believe that we are deriving reliable parameters so let us 
determine if at least we are being i n t e r n a 11 y consistent. For the e f $f$ e c $t$ i ve temperatures most analyses quote an internal uncertainty of $\pm 200 \mathrm{~K}$ which is equivalent to the t o t a l r a n g e observed for this star. The derived temperatures are concentrated in the 4580 - $4620 \mathrm{~K}$ range indicating $t h$ a $t h e$ temperature determination is not the most severe constraint on the accuracy of the $[\mathrm{Fe} / \mathrm{H}]$ ratio.

A consistency check which can be made is to plot effective temperature versus $\log g$ (see Figure 7). Methods commonly used to determine gravities (ionization balance and assumed mass and luminosity) demand that as

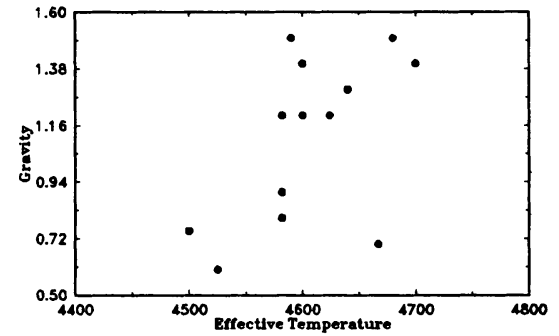

Figure 7: Effective temperature versus gravity for individual determinations for HD 122563.

the temperature decreases the gravity also decreases. This is indeed the case demonstrated in Figure 7 and the dependence is quite steep. Since the dominant method of gravity determination for this star has been ionization balance which is very sensitive to temperature in this regime, the steep dependence is not unexpected. Thus we can have some consolation in this data in finding that we are at least being internally consistent insofar as the gravity is concerned. 
If the effective temperature is relatively well constrained, and the gravity determinations are consistent with the assigned effective temperatures, why do the derived $[\mathrm{Fe} / \mathrm{H}]$ ratios spread out over $0.6 \mathrm{dex}$ - a factor of 4 ? Could the culprit be in the observational data or in the iron oscillator strengths? Obviously much progress has been made on these fronts over the past 30 years but HD 122563 is a bright star and "good" data has always been available for analysis. It is also unlikely that errors in oscillator strengths could lead to a factor of 4 difference in the abundance. Bad oscillator strengths are uncertain at the 50 percent level, not at the observed differences. So what is the problem? The most likely explanation lies in the coupling of $\mathrm{T}_{\text {eff }}-\log \mathrm{g}$ and $[\mathrm{Fe} / \mathrm{H}]$ as demonstrated for this star in Figure 8 . A regression plane fits the data very well and indicates that systematic trends dominate the abundance analyses. From Figure 7 we know that high gravities are associated with high temperatures and the converse also holds. Figure 8 shows that high abundances are associated with high gravity-temperature pairs and that the low ratios are associated with the low temperature-gravity pairs. The deviations from the regression plane are on the order of 0.1 dex - comparable to the expected internal uncertainty in the analysis (typically \pm 0.2 dex). Thus the secret to obtaining reliable $[\mathrm{Fe} / \mathrm{H}]$ values ultimately lies in our ability to determine the gravity of our object stars. Unfortunately, this is one of the most difficult tasks in stellar physics.

\section{The Globular Clusters}

Another way to evaluate the reliability of $[\mathrm{Fe} / \mathrm{H}]$ ratios is to examine values derived for homogeneous samples. The globular cluster systems are excellent examples of this and Figure 9 shows $[\mathrm{Fe} / \mathrm{H}]$ ratios determined for numerous globular clusters. All values shown

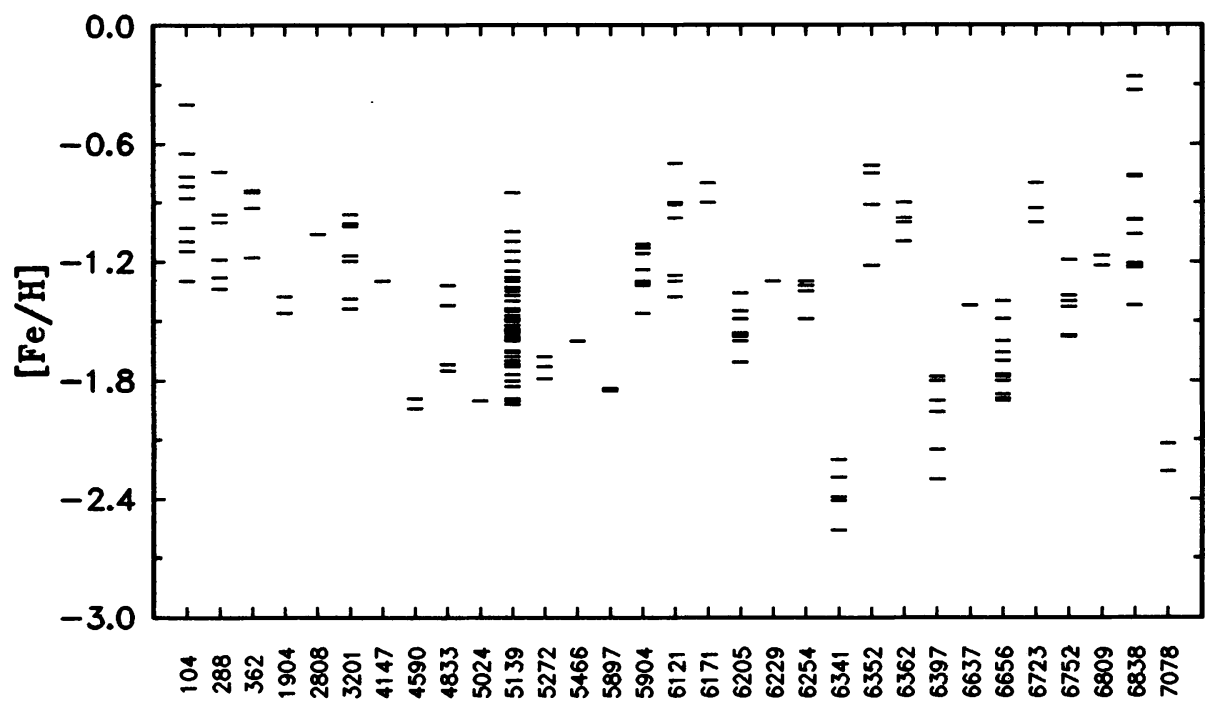

Figure 9: $[\mathrm{Fe} / \mathrm{H}]$ ratios for individual stars in globular clusters. 
are for individual stars - multiple observations have been averaged. $\omega$ Cen (NGC 5139) and M 71 (NGC 6838) have a real spread in [Fe/H] (Pilachowski 1984, Caldwell and Dickens 1988, Paltoglou and Norris 1989) but the remainder are presumably homogeneous. The abundance spread evidenced in these clusters is typically 0.3 to 0.5 dex, equivalent to the total spread observed in HD 122563. Once again, the most likely culprit is differences in $T_{\text {eff }}$ - $\log \mathrm{g}$ scales between the various analyses. For clusters analyzed within the same work the spread is usually substantially lower: 0.2 dex (Gratton 1987, 1989).

\section{6. $[\mathrm{O} / \mathrm{Fe}]$ Ratios}

One of the most striking results in the past decade in the field of metal-poor stars is the demonstration of the dependence of $[\mathrm{O} / \mathrm{Fe}]$ on $[\mathrm{Fe} / \mathrm{H}]$ (or alternately, $[\mathrm{Fe} / \mathrm{O}]$ on $[\mathrm{O} / \mathrm{H}]$ ). This subject has been discussed extensively (see Wheeler, Sneden, and Truran 1989 and Abia and Rebolo 1989: Figure 2 of Wheeler, Sneden, and Truran shows the relation as do Figures 2, 5, and 6 of Abia and Rebolo). There are two "independent" atomic features available for the oxygen abundance determination: the $[O \mathrm{I}]$ lines and the $\mathrm{O} I 7774 \AA$ triplet. To determine the $[\mathrm{O} / \mathrm{Fe}]$ ratio the observation data necessary is demanding: at $T_{\text {eff }}=4500 \mathrm{~K}$, $\log \mathrm{g}=1.0$, and $[\mathrm{O} / \mathrm{H}]=-2.1$, the equivalent width of $[\mathrm{O} \mathrm{I}] 6300 \AA$ is $9.8 \mathrm{~m} \AA$ while $\mathrm{O} \mathrm{I}$ 7771 is $2.5 \mathrm{~m} \AA$. Additionally, as pointed out by Wheeler, Sneden, and Truran, it is necessary to document the agreement of the oxygen abundance from both features. Their parameter sensitivity is very different and greater confidence can be placed in the claimed trends if it can be shown that both features yield the same ratio with respect to iron. This has yet to be accomplished.

A potential problem which has not been addressed is demonstrated in Table 3 where synthetic $[\mathrm{O} / \mathrm{Fe}]$ ratios are given as a function of $\mathrm{T}_{\text {eff }}$ and $\log \mathrm{g}$ for both $[\mathrm{O} \mathrm{I}]$ and $\mathrm{O} \mathrm{I}$ (within the table $[\mathrm{O} \mathrm{I}]$ is first, $\mathrm{O}$ I second). The values were derived such that $[\mathrm{O} / \mathrm{Fe}]=0.51$ at $\mathrm{T}_{\text {eff }}$ $=4500$ and $\log g=1.0$. To understand the behavior in Table 3: 1) At constant gravity and increasing temperature: $[\mathrm{Fe} / \mathrm{H}]$ increases; $[\mathrm{O} / \mathrm{H}]$ from $[\mathrm{O} I]$ remains constant, and $[\mathrm{O} / \mathrm{H}]$ from $O$ I decreases, and 2) at constant temperature and increasing gravity: $[\mathrm{Fe} / \mathrm{H}]$ decreases; $[\mathrm{O} / \mathrm{H}]$ from $[\mathrm{O} \mathrm{I}]$ increases, and $[\mathrm{O} / \mathrm{Fe}]$ from $\mathrm{O} \mathrm{I}$ increases.

\begin{tabular}{||c|c|c|c|c|c||}
\hline \multicolumn{7}{|c||}{ Table 3 } \\
\hline$T_{\text {eff }}$ & $\log \mathrm{g}=0.5$ & $\log \mathrm{g}=0.75$ & $\log \mathrm{g}=1.0$ & $\log \mathrm{g}=1.25$ & $\log \mathrm{g}=1.5$ \\
\hline \multirow{2}{*}{4300} & +0.48 & +0.64 & +0.78 & +0.90 & +1.01 \\
& +0.77 & +0.93 & +1.08 & +1.21 & +1.33 \\
\hline \multirow{2}{*}{4400} & +0.33 & +0.49 & +0.64 & +0.77 & +0.89 \\
& +0.47 & +0.64 & +0.79 & +0.93 & +1.05 \\
\hline \multirow{2}{*}{4500} & +0.22 & +0.37 & +0.51 & +0.64 & +0.78 \\
& +0.20 & +0.36 & +0.51 & +0.65 & +0.77 \\
\hline \multirow{2}{*}{4600} & +0.15 & +0.27 & +0.41 & +0.54 & +0.66 \\
& -0.04 & +0.10 & +0.25 & +0.38 & +0.51 \\
\hline \multirow{2}{*}{4700} & +0.09 & +0.21 & +0.33 & +0.45 & +0.57 \\
& -0.28 & -0.13 & +0.00 & +0.14 & +0.26 \\
\hline
\end{tabular}


From the data given in Table 3 caution is warranted in the discussion of $[\mathrm{O} / \mathrm{Fe}]$ ratios, and in particular, in the comparison of $[\mathrm{O} / \mathrm{Fe}]$ ratios of red-giants from different sources. The uncertainties in the stellar parameters are evident in the $[\mathrm{O} / \mathrm{Fe}]$ ratios in all cases as iron and oxygen do not share the same parameter sensitivity. Further, even if the ratios agree from [O I] and $\mathrm{O} I$, that does not mean that the ratio is correct (or that the parameters are correct) - see the middle row of Table 3. If the ratios do not agree - do not necessarily assume that the problem is non-LTE. Small adjustments to the parameters may be able to rectify the answers. Lastly, perhaps the best way to determine $[\mathrm{O} / \mathrm{Fe}]$ ratios is to use the [O I] lines and use an ionization balance to determine the gravity. In that case the temperature-gravity determination moves along the diagonal where the $[\mathrm{O} / \mathrm{Fe}]$ ratio remains relatively constant.

\section{Concluding Remarks}

The accuracy of any abundance or abundance ratio ultimately depends on the accuracy of the stellar parameters. Investigation of the systematics of the $[\mathrm{Fe} / \mathrm{H}]$ ratios in metal-poor stars indicates that a significant fraction, if not the bulk, of the systematic uncertainties in the $[\mathrm{Fe} / \mathrm{H}]$ ratio can be ascribed to the stellar parameters. This uncertainty also extends to discussion of such abundance ratios as $[\mathrm{O} / \mathrm{Fe}]$. A corollary of this conclusion is that data quality is not the overriding consideration in the determination of $[\mathrm{Fe} / \mathrm{H}]$ ratios. As long as there are no systematic errors in the equivalent widths (begging the question of which scale is correct!) the $[\mathrm{Fe} / \mathrm{H}]$ ratio will be well constrained so long as the uncertainty in the mean is $\leq 0.05$ dex (which means that for 100 lines that the standard deviation can be up to 0.5 dex!).

It is my opinion that the larger problem with the metal-poor analyses lies in the gravities. This is not a problem specific to metal-poor stars but one which afflicts all analyses based on theoretical model-atmospheres. What should be the next step in our work on metal-poor stars? I cannot say that we should stop analyzing stars until the gravity problem is solved that would be counterproductive. The solution may very well lie within those analyses. I merely urge all of us to recognize these problems and address them in the best fashion possible.

\section{Acknowledgements}

The references for the individual analyses, the results of those analyses, and the average values are too lengthy for publication in this review. This material is available upon request from the author. Requests should be by e-mail to luck@cwast3.astr.cwru.edu. This work has been supported by NSF grant 89-13092 at Case Western Reserve University.

\section{REFERENCES}

Abia, C., and Rebolo, R. 1989, Ap. J. 347, 186.

Caldwell, S. P., and Dickens, R. J. 1988, M. N. R. A. S. 233, 367.

Cayrel de Strobel, G., Bentolila, C., Hauck, B., and Duguennoy, A. 1985, Astr. Ap. Suppl. 59, 145.

Cayrel de Strobel, G., Spite, M., and Lloyd Evans, T. (eds.) 1988, The abundance spread within Globular Clusters: spectroscopy of individual stars (IAU JCM 5 and CM37/3), Paris Observatory, Paris.

Gustafsson, B., Bell, R. A., Eriksson, K., and Nordlund, A. 1975, Astr. Ap., 42, 407.

Gratton, R. G. 1987 Astr. Ap. 179, 181. 
1989 Astr. Ap. $211,41$.

Paltoglou, G., and Norris, J. E. 1989, Ap. J. 336, 185.

Pilachowski, C. A. 1984, Ap. J. 281, 614.

Spite, M., and Spite, F. 1985, A.R.A.A. 23, 225.

Wheeler, J. C., Sneden, C., and Truran, J. W. 1989, A.R.A.A. 27, 279. 